phase were decreased appetite, insomnia, affect lability, upper abdominal pain, mood swings, and headache.

CONCLUSIONS: AMPH EROS was effective in reducing symptoms or ADHD from 1 to 13 hours after dosing. Adverse events reported were consistent with those of other amphetamine products.

Funding Acknowledgements: This study was supported by Tris Pharma, Inc.

89

\section{A Novel, Modified-Release Drug Delivery Technology Containing Amphetamine-Ion Exchange Complexes}

Barry K. Herman, MD, $M M M^{\prime}$; Thomas R. King, MS, $\mathrm{MPH}^{\prime}$; Judith C. Kando, PharmD, BCPP'; and Antonio Pardo, $M D^{\prime}$

${ }^{1}$ Tris Pharma, Inc., Monmouth Junction, NJ

ABSTRACT: The proprietary immediate and extended drug delivery technology LiquiXR ${ }^{\mathrm{TM}}$ utilizes an ion-exchange resin that complexes with amphetamine or any other active moiety that can be protonated and is water-soluble. The active drug product forms a complex with ionexchange polymers contained in the resin, which is then formed into micron-sized particles. Some of these particles are coated with an aqueous, $\mathrm{pH}$-independent polymer designed to provide immediate or sustained release of active drug product. The polymer coating applied to the ion-exchange resin particles is of varying thickness, allowing for programmed, extended release of active drug product. Solid, coating-free particles provide for immediate release of active drug product.

The micron-sized particles are formulated into an appropriate dosage form (solid or chewable tablet, liquid suspension, orally disintegrating tablet, film, or capsules). Active drug product is subsequently released from the dosage form in millions of particles, with the release driven by a combination of ion exchange and diffusion. After drug release, the ion-exchange resin is excreted in the feces.

The release characteristics of LiquiXR ${ }^{\mathrm{TM}}$ are programmable and allow for a customized, sustained release of active drug product for up to 24 hours post-dose. Mechanistically, drug particles enter the gastrointestinal tract. As positively-charged ions from gastrointestinal (GI) fluids diffuse across the coating, ionically-charged drug product diffuses through the coating and into the GI fluids for absorption. As the coating is of variable thickness, some drug product takes longer to diffuse and absorb, providing for the programmable delayed drug release characteristic.
The LiquiXR ${ }^{\mathrm{TM}}$ drug delivery technology is utilized in Dyanavel ${ }^{\circledR}$ XR (amphetamine extended-release oral suspension; AMPH EROS), which is indicated for the treatment of attention-deficit hyperactivity disorder. It comprises $2.5 \mathrm{mg} / \mathrm{mL}$ amphetamine base complexed with LiquiXR technology to provide an immediate release component followed by an extended-release profile. The efficacy of AMPH EROS was established in children ages 6 to 12 years in a Phase 3, placebocontrolled laboratory classroom study. In that study, attention-deficit/hyperactivity disorder (ADHD) symptoms in children on an individually optimized dose of amphetamine (range 10-20 mg/day) were statistically significantly improved compared with symptoms in children treated with placebo. For children treated with AMPH EROS, onset of effect was demonstrated at 1 hour after dosing, and efficacy was observed through 13 hours post-dose. The effect size was comparable to effect sizes demonstrated for other psychostimulants tested in studies using a similar design. The efficacy data reported for AMPH EROS provides an excellent example of the potential utility and clinical application for other active drug products requiring an immediate release and extended release profile.

Funding Acknowledgements: This work was funded by Tris Pharma, Inc.

\section{2}

\section{Effects of Smartphone Coaching Intervention on Dietary Intake for Bariatric Surgery Candidates: A Pilot Randomized Controlled Trial}

Youngjung Kim, MD, PhD'; Robyn Sysko, $P h D^{2}$; Andreas Michaeledes, $P h D^{3}$; Tatiana Ramos, $P h D^{3}$; and Tom Hildebrandt, PsyD ${ }^{4}$

${ }^{1}$ Department of Psychiatry, Icahn School of Medicine at Mount Sinai, New York, NY

${ }^{2}$ Department of Psychiatry, Icahn School of Medicine at Mount Sinai, New York, NY

${ }^{3}$ Noom, Inc., New York, NY

${ }^{4}$ Department of Psychiatry, Icahn School of Medicine at Mount Sinai, New York, NY

ABSTRACT: Introduction: Bariatric surgery outcomes are variable, often with suboptimal weight loss and/or weight relapse. Smartphone coaching applications offer a potential window of affecting behavioral change to improve outcomes in a widespread and cost-effective way, but clinical efficacy is unknown. In phase I of this pilot study, we investigated effects of pre-surgical treatment with a mobile coaching platform Noom Coach for Bariatric Health.

METHODS: Forty adult candidates $(82.5 \%$ female $)$ for bariatric surgery were recruited for pilot randomized 